\title{
Physical Activity and Sedentary Behaviour in Croatian Preschool Children: A Population- Based Study
}

\author{
Maja Vukelja', Dragan Milanovic' ${ }^{1}$, Sanja Salaj' \\ Affiliations: 'University of Zagreb Faculty of Kinesiology, Zagreb, Croatia
}

Correspondence: M. Vukelja, University of Zagreb, Faculty of Kinesiology, Horvacanski zavoj 15, 10000 Zagreb, Croatia. E-mail: maja.vukelja@kif.hr

\begin{abstract}
This study aimed to determine the differences in physical activity and sedentary behaviour in preschool children living in different geographical regions. Preschool children $(\mathrm{N}=1625)$ and their parents from different parts of Croatia, from urban and rural settlements, participated in this cross-sectional, population-based study. Parents completed the Netherlands Physical Activity Questionnaire (NPAQ), a measure of physical activity and sedentary behaviour in children. The main results of this study show differences in physical activity and sedentary behaviour in preschool children living in four geographical regions in Croatia $(F=4.45 ; p<0.01)$. The least physically active are children from a continental area that gravitates to the capital city, while the most active are children from a southern coastal region. Sedentary behaviour is the greatest in the rural eastern Croatian continental region. Higher physical activity and lower sedentary activities in young children living in coastal compared to continental regions show possible specific advantages of Mediterranean climate in general. The practical importance of information obtained in this study is a need for a specific intervention strategy for improving physical activity in continental preschool institutions.
\end{abstract}

Keywords: kindergarten, Mediterranean, screen-time, rural, urban

@MJSSMontenegro

PHYSICAL ACTIVITY AND SEDENTARY BEHAVIOUR IN CROATIA

http://mjssm.me/?sekcija=article\&artid=229

Cite this article: Vukelja, M., Milanovic, D., \& Salaj, S. (2022). Physical Activity and Sedentary Behaviour in Croatian Preschool Children: A Population-Based Study. Montenegrin Journal of Sports Science and Medicine, 11 (1), $37-42$. https://doi.org/10.26773/mjssm.220304

\section{Introduction}

Physical inactivity is recognized as the biggest public health problem of the $21^{\text {st }}$ century. To prevent obesity and other non-communicable diseases, physical activity, increased physical fitness, and reduced sedentary behaviour are crucial. Various public health actions are being taken to raise awareness of the importance of physical activity. Public health initiatives to promote physical activity use a mul- tisector, multisystem approach. Schools and sports clubs play a major role in these initiatives by providing physical activity for children and offering programmes to develop knowledge and skills for healthy and active living habits (Investments that Work for Physical Activity, 2012).

Health institutions worldwide have made recommendations regarding the necessary daily amount of physical activity for children. For preschool children, Canada 
(Tremblay et al., 2012), the United Kingdom (Department of Health, 2011), and Australia (Department of Health and Aging 2010) recommend up to 180 minutes of physical activity per day. National recommendations for Croatia proposed by Jurakic and Pedisic (2019) are in line with the latest World Health Organization (WHO) guidelines for preschool children (WHO, 2019). It is generally recommended that at least 60 minutes out of these 180 minutes of various physical activities are spent in moderate-to-vigorous play.

In addition to the physical activity guidelines, some countries have also set guidelines for sedentary behaviour in preschool children. Children from one to five years of age should not engage in sedentary activities for more than one hour a day (excluding sleep time). Furthermore, oneto two-year-old children should not have any screen time (TV, computer, or mobile phone), while for children aged three and four, this time should be limited to the maximum of one hour per day. If children spend time in sedentary activities, it is recommended that this time be devoted to reading and storytelling or other types of intellectual and fine motor development activities (WHO, 2019; Jurakic\& Pedisic, 2019).

Published research on the level of physical activity and sedentary behaviour in preschool children shows that the physical activity is generally lower and that the sedentary behaviour is generally higher than the abovementioned recommended time values. For example, according to Tucker's review (2008) of studies from seven countries (four of which European), involving 10,316 preschool children aged 2 to 6 , almost half of the children did not reach the recommended daily level of physical activity for their respective age. In contrast, a study of preschool children conducted in Britain by Hall and co-authors (2018) found that $80.30 \%$ of them did achieve the recommended daily level of physical activity. Another study, by Barbosa and Oliveira (2016), showed that preschool children spend much time in sedentary activities (even during their stay in kindergartens) and that sedentary activities are more frequent when children are indoors (up to $94 \%$ of the time) compared to when they are outdoors (Barbosa \& Oliveira, 2016).

To successfully implement a physical activity promotion system in a specific setting (country, region, culture), it is important to understand the factors on which the physical activity depends. These factors vary from childhood to adulthood. According to relevant research, the level of physical activity of preschool and early school-age children largely depends on gender, age, self-confidence in movement, physical engagement in certain situations, family support, history of premature birth, kindergarten environment and the father's body mass index (BMI) (Bauman et al., 2012; Finn et al., 2002; Barbosa \& Oliveira, 2016). Furthermore, it seems that as the children grow older, the time spent in medium- to high-intensity physical activity decreases, while the time spent in low- to medium-intensity physical activity increases (Barbosa \& Oliveira, 2016). This shows that growing up weakens the intensity of physical activity gradually, and it is thus very important to motivate and involve preschool children in various forms of physical activity from an early age. Doing so could be seen as the responsibility of a variety of educational and health institutions.

According to the World Health Organization data for the Republic of Croatia (WHO, 2018), 88\% of children aged 8 meet the recommended values of physical activity (60 minutes), but this percentage decreases to only $19 \%$ in adolescents (WHO, 2018). Therefore, one would expect preschool children to meet the recommended level of physical activity, but the study by Petric et al. (2019) conducted on a small sample of Croatian preschool children found that that was not the case. These children did not meet the daily recommended level of physical activity (180 minutes). Although the physical activity of school-aged children in Croatia has been studied before (Janssen et al., 2005), this study presents physical activity and sedentary behaviour in Croatian preschool children of three to six years of age.

This study aims to determine the differences in physical activity levels and sedentary behaviour in preschool children of different ages, sexes, and places of residence in Croatia.

\section{Methods}

A total of 1,625 preschool boys and girls aged 3 to 6 from different parts of Croatia participated in this study (Table 1). Since this research was a part of a larger study, the sample size was larger than the minimal recommended (Raosoft sample size calculator). The average age of the participants was 5.2 years. The Republic of Croatia is divided into four geographically and economically different macro-regions (the largest is the Central or Zagreb macro-region (50\% of the population), followed by the Dalmatian or Split macro-region, the Eastern Croatian or Osijek macro-region and the Northern Croatian coast or Rijeka macro-region). Based on the official 2011 census of the State Bureau of Statistics (the total of 166,439 children) and the proportion of the preschool children population in the population of each region (48\% for Zagreb macro-region, 22\% for Rijeka macro-region, $18 \%$ for Osijek macro-region and $12 \%$ for Split macro-region), the size of the sample for each region was calculated. Based on the initial proportions, the kindergartens included in the study were randomly selected from the official governmental list. Therefore, all children from these kindergartens were included in the measurements.

\section{Measurements}

This study was conducted from September 2018 to May 2019 as a part of a larger research project on motor skills in preschool children in Croatia. The physical activity and sedentary activities of preschool children were evaluated by their parents using "The Netherlands Physical Activity Questionnaire” (NPAQ) (Janz et al., 2005), which was adapted to the Croatian language (Kezic \& Miletic, 2014; Culjak et al., 2014). NPAQ is a Likert-type questionnaire consisting of seven questions about a child's physical activity scaling responses on a scale of 1 to 5 . It is a simple and practical measure of everyday physical activity preferences in young children, which has moderate to good reliability (Janz et al., 2005). Physical activity results are reported as an average score of seven questions, while sedentary behaviour (average hours spent watching TV, using computers and mobile phones) is expressed in minutes per day (Janz et al., 2005). Questionnaires were filled out by mothers (83.51\% of the cases), fathers (15.02\% of the cases) or others (i.e., both mother and father, grandmother, foster mother or not specified) (1.47\% of the cases). 
Table 1. Sample distribution according to regions, gender and age (number (percentage)

\begin{tabular}{|c|c|c|c|c|c|c|c|c|c|}
\hline & \multicolumn{2}{|c|}{ Zagreb } & \multicolumn{2}{|c|}{ Rijeka } & \multicolumn{2}{|c|}{ Osijek } & \multicolumn{2}{|c|}{ Split } & \multirow{2}{*}{ Total } \\
\hline & Urban & Rural & Urban & Rural & Urban & Rural & Urban & Rural & \\
\hline $\mathrm{N}$ & $\begin{array}{c}480 \\
(29.54 \%)\end{array}$ & $\begin{array}{c}357 \\
(21.97 \%)\end{array}$ & $\begin{array}{c}152 \\
(9.35 \%)\end{array}$ & $\begin{array}{c}169 \\
(10.4 \%)\end{array}$ & $\begin{array}{c}147 \\
(9.05 \%)\end{array}$ & $\begin{array}{c}102 \\
(6.28 \%)\end{array}$ & $\begin{array}{c}139 \\
(8.55 \%)\end{array}$ & $\begin{array}{c}99 \\
(6.09 \%)\end{array}$ & 1625 (100\%) \\
\hline Male & $\begin{array}{c}263 \\
(16.19 \%)\end{array}$ & $\begin{array}{c}191 \\
(11.75 \%)\end{array}$ & 76 (4.68\%) & $83(5.11 \%)$ & 76 (4.68\%) & 45 (2.77\%) & 73 (4.49\%) & 50 (3.08\%) & $\begin{array}{c}857 \\
(52.74 \%)\end{array}$ \\
\hline Female & $\begin{array}{c}217 \\
(13.35 \%)\end{array}$ & $\begin{array}{c}166 \\
(10.22 \%)\end{array}$ & $76(4.68 \%)$ & $86(5.29 \%)$ & 71 (4.37\%) & 57 (3.51\%) & $66(4.06 \%)$ & 49 (3.02\%) & $\begin{array}{c}788 \\
(48.49 \%)\end{array}$ \\
\hline Age 3 & $\begin{array}{c}92 \\
(5.66 \%)\end{array}$ & $\begin{array}{c}43 \\
(2.65 \%)\end{array}$ & $\begin{array}{c}32 \\
(1.97 \%)\end{array}$ & $\begin{array}{c}42 \\
(2.59 \%)\end{array}$ & $\begin{array}{c}27 \\
(1.66 \%)\end{array}$ & $\begin{array}{c}13 \\
(0.8 \%)\end{array}$ & $\begin{array}{c}31 \\
(1.91 \%)\end{array}$ & $\begin{array}{c}18 \\
(1.11 \%)\end{array}$ & $\begin{array}{c}298 \\
(18.34 \%)\end{array}$ \\
\hline Age 4 & $\begin{array}{c}124 \\
(7.63 \%)\end{array}$ & $\begin{array}{c}92 \\
(5.66 \%)\end{array}$ & $\begin{array}{c}40 \\
(2.46 \%)\end{array}$ & $\begin{array}{c}61 \\
(3.75 \%)\end{array}$ & $\begin{array}{c}42 \\
(2.59 \%)\end{array}$ & $\begin{array}{c}20 \\
(1.23 \%)\end{array}$ & $\begin{array}{c}48 \\
(2.95 \%)\end{array}$ & $\begin{array}{c}31 \\
(1.91 \%)\end{array}$ & $\begin{array}{c}458 \\
(28.19 \%)\end{array}$ \\
\hline Age 5 & $\begin{array}{c}86 \\
(5.29 \%)\end{array}$ & $\begin{array}{c}88 \\
(5.42 \%)\end{array}$ & $\begin{array}{c}49 \\
(3.02 \%)\end{array}$ & $\begin{array}{c}41 \\
(2.52 \%)\end{array}$ & $\begin{array}{c}28 \\
(1.72 \%)\end{array}$ & $\begin{array}{c}28 \\
(1.72 \%)\end{array}$ & $\begin{array}{c}34 \\
(2.09 \%)\end{array}$ & $\begin{array}{c}24 \\
(1.48 \%)\end{array}$ & $\begin{array}{c}378 \\
(23.26 \%)\end{array}$ \\
\hline Age 6 & $\begin{array}{c}164 \\
(10.09 \%)\end{array}$ & $\begin{array}{c}131 \\
(8.06 \%)\end{array}$ & $\begin{array}{c}31 \\
(1.91 \%)\end{array}$ & $\begin{array}{c}25 \\
(1.54 \%)\end{array}$ & $\begin{array}{c}50 \\
(3.08 \%)\end{array}$ & $\begin{array}{c}38 \\
(2.34 \%)\end{array}$ & $\begin{array}{c}26 \\
(1.6 \%)\end{array}$ & $\begin{array}{c}26 \\
(1.6 \%)\end{array}$ & $\begin{array}{c}491 \\
(30.22 \%)\end{array}$ \\
\hline
\end{tabular}

\section{Data analysis}

Statistical analyses were performed using TIBCO Statistica v.13 software (TIBCO Statistica Inc, OK, USA). Differences in the degree of physical activity and sedentary behaviour of the preschool children of different ages and from different regions were determined using the analysis of variance (one-way and two-way ANOVA) and the unequal N HSD post hoc test. Statistical significance was set to $\mathrm{p}<0.05$.

\section{Results}

The physical activity of preschool children significantly differs among the four macro-regions in Croatia $(\mathrm{F}=9.41$; $\mathrm{p}<0.01$ ) (Table 1). There were no significant differences in the preschool children's physical activity in different types of settlements $(\mathrm{F}=0.60 ; \mathrm{p}=0.44)$. There was no significant interaction between factors settlement type and region.

The level of the physical activity of the preschool children from rural settlements of the Central (Zagreb) macro-region (3.46) is significantly lower than the level of the physical activity of the preschool children from rural settlements of the Dalmatian (Split) macro-region (3.73; $\mathrm{p}<0.05)$.

Two-way ANOVA showed that sedentary behaviour is significantly different in children from the different types of settlements in Croatia $(\mathrm{F}=15.14 ; \mathrm{p}<0.01)$ and different mac-

Table 2. Differences in the level of physical activity and sedentary behaviour of preschool children in different Croatian macro-regions (MEAN $\pm \mathrm{SD}$ )

\begin{tabular}{ccccc}
\hline & \multicolumn{2}{c}{ Physical activity } & \multicolumn{2}{c}{ Sedentary behaviour } \\
\cline { 2 - 5 } & Urban settlements & Rural settlements & Urban settlements & Rural settlements \\
\hline Zagreb & $3.54 \pm 0.59$ & $3.46 \pm 0.61$ & $132.69 \pm 75.44$ & $145.54 \pm 69.21$ \\
Rijeka & $3.59 \pm 0.49$ & $3.63 \pm 0.54$ & $112.22 \pm 79.44^{*} \dagger$ & $118.68 \pm 74.00^{*} \dagger$ \\
Osijek & $3.62 \pm 0.56$ & $3.66 \pm 0.53$ & $129.11 \pm 58.18$ & $150.69 \pm 75.52$ \\
Split & $3.65 \pm 0.53$ & $3.73 \pm 0.54^{*}$ & $112.68 \pm 55.89^{*} \dagger$ & $134.00 \pm 72.53$ \\
\hline
\end{tabular}

Note. * - significantly different from rural settlements in the Zagreb macro-region $(p<0.01)$, $\uparrow$-significantly different from rural settlements in the Osijek macro-region $(p<0.01)$.

ro-regions $(\mathrm{F}=10.65 ; \mathrm{p}<0.01)$ (Table 2$)$. There was no significant interaction between factors settlement type and region. Sedentary behaviour in the preschool children from the rural settlements of the Zagreb macro-region (145.54) and the rural settlements of the Eastern Croatian (Osijek) macro-region (150.69) are significantly higher than the sedentary behaviour of the preschool children from the urban $(112.22 ; \mathrm{p}<0.01)$ and the rural $(118.51 ; \mathrm{p}<0.01)$ settlements of the Northern Croatian coast (Rijeka) and the children from the Dalmatian urban settlements $(112.68 ; \mathrm{p}<0.01)$. There were no differences in sedentary behaviour within one specific macro-region. From all rural settlements, sedentary behaviour is the lowest on the Northern Croatian coast: significantly lower in Zagreb and the Eastern Croatian region $(\mathrm{p}<0.05)$.

Table 3. Differences in the level of physical activity and sedentary activities in preschool children in different age groups (MEAN \pm SD)

\begin{tabular}{ccc}
\hline Age & Physical activity & Sedentary behaviour \\
\hline 3 & $3.66 \pm 0.55$ & $102.36 \pm 60.15^{*} \dagger$ \\
4 & $3.61 \pm 0.57$ & $116.58 \pm 68.71^{*} \dagger$ \\
5 & $3.54 \pm 0.58$ & $141.31 \pm 74.32$ \\
6 & $3.56 \pm 0.58$ & $150.75 \pm 77.22$ \\
\hline
\end{tabular}

Note. ${ }^{*}$ - significantly different from five-year-old children at $\mathrm{p}<0.01, \uparrow$ - significantly different from six-year-old children at $\mathrm{p}<0.01$. 
Physical activity was not significantly different in preschool children of different ages $(\mathrm{F}=2.61 ; \mathrm{p}=0.501)$, although average values show higher physical activity in younger compared to older children (Table 3). Significant differences were found in the amount of sedentary activities of preschool children of different age groups $(\mathrm{F}=30.35 ; \mathrm{p}<0.01)$. The three-year-olds (102.36; $\mathrm{p}<0.01)$ and the four-year-olds (116.58; $\mathrm{p}<0.01)$ spent significantly less time in sedentary activities compared to the five-year-olds (141.31) and the six-year-olds (150.75).

Furthermore, physical activity differed significantly with respect to gender $(\mathrm{F}=43.57 ; \mathrm{p}<0.01)$. Preschool boys $(3.66 \pm 0,58)$ were more physically active than preschool girls $(3.48 \pm 0,55 ; \mathrm{p}<0.01)$. We also found significant differences in the sedentary behaviour of boys and girls $(\mathrm{F}=18.65$; $\mathrm{p}<0.01)$. Preschool boys spent more time in sedentary activities $(138.92 \pm 77,82)$ than preschool girls did $(123.67 \pm 64,83$; $\mathrm{p}<0.01)$.

\section{Discussion}

This study reveals that children from the Central region, i.e., the continental area surrounding the capital city, are the least physically active, while the children from the rural settlements in the Dalmatian coastal region are the most active of all the preschoolers' in Croatia. Although there are differences in the level of physical activity in the children of the different geographical regions of Croatia, there are no differences in physical activity in children that could be attributed to the urban or rural setting in general. Physical activity is higher in boys compared to girls, and the tendency of lower physical activity is observable in older compared to younger preschool children.

The average physical activity results in children for almost all regions can be characterized as a high level of physical activity (results from 3.5 to 5): $53.8 \%$ of the total number of preschool children included in this research were categorized as highly active, meaning that they reached a satisfying level of physical activity. The only exception was the rural part of the Zagreb macro-region. The preschool children of this region were only moderately physically active (3.46) (results from 2.50 to 3.49). Observing the physical activity across the macro-regions (the sum of urban and rural settlements of a particular macro-region), it is evident that preschool children from the Dalmatian macro-region are the most active, while the preschool children from the Central macro-region are the least active. We can assume that various factors, such as different climates (continental vs Mediterranean), different lifestyles, and socioeconomic factors, are responsible for the differences in the physical activity in the children of different regions.

Furthermore, it is must be emphasized that preschool children from the coastal parts spend less time in sedentary activities, such as watching TV screens and playing computer games, compared to the preschool children from the continental parts. Bergman Markovic et al. (2011) found a greater prevalence of cardiovascular risk factors in adults of the continental region compared to the adults living in the Mediterranean parts of Croatia. They suggested that the likely explanation is in their different lifestyles. Having the long-term consequences of physical inactivity and increased sedentary behaviour in mind (Lavie et al., 2019), it is a worrying possibility that cardiovascular risk factors are region-specific.

Similar to previous studies examining gender differences in physical activity in children, preschool boys' physical activity levels were higher than those of preschool girls (Finn et al., 2002; Cardon et al., 2008; Sallis et al., 2000). A study conducted in the USA, using the same questionnaire (NPAQ), on a sample of children under 10 years of age showed that boys are more active (3.6 - high physical activity level) than girls (3.3 - medium level of physical activity) (Janz et al., 2005). These results are consistent with our study. Croatian boys have achieved a high physical activity level (3.66) compared to girls who have shown a medium level of physical activity (3.48).

This research also shows the differences in the duration of sedentary activities (watching TV and playing computer games) of the preschool children in the four Croatian regions. Those differences are the biggest in rural settlements. Sedentary behaviour is most prominent in the rural eastern continental region. In contrast, the preschool children from the urban settlements of both northern and southern coastal regions spend the least time in sedentary activities. On average, the preschool children from the rural settlements in the Eastern continental macro-region engage in sedentary behaviour for 2.5 hours per day, while the preschool children from the urban settlements in the two coastal regions engage in sedentary behaviour for less than two hours a day. Comparing different countries, Santaliestra-Pasias et al. (2013) were able to show regional as well as national differences. The data from their study (Santaliestra-Pasias et al., 2013) indicates that, compared to Italian, Estonian, Belgian, German, Swedish, Hungarian and Spanish children, Cypriot children spent the largest amount of time in sedentary activities such as watching television, DVDs, and other video content. Compared to the days of the week, there was a drastic increase in sedentary activities of this type during weekends. For this reason, particular attention should be given to the physical activity organized by both professionals and parents during the weekend periods.

The findings of this study indicate that children of different ages and genders differ significantly with respect to time spent in sedentary activities. It should be noted that the time spent in sedentary activities gradually increases with age, which is a very serious problem considering that the preschoolers have not yet reached the age at which a greater increase in sedentary behaviour is expected (school-age). Although more active than girls, boys have higher sedentary behaviour. This finding is in line with the abovementioned research of Santaliestra-Pasias et al. (2013), which showed that boys spend more time in sedentary activities than girls in the majority of the countries that were part of the study. In contrast to this, a US study of preschool children revealed that girls spend more time in sedentary activities (120 $\mathrm{min}$ ) than boys do (108 $\mathrm{min}$ ) (Janz et al., 2005). This shows that even if one child can be highly physically active for a while, he or she can then exhibit sedentary behaviour for an equal or longer amount of time playing computer games and watching television. The average duration of sedentary activities for all children in this research was 131.55 minutes, which exceeds given recommendations restricting daily sedentary behaviour to under one hour (WHO, 2019; Department of Health and Aging, 2010) as well as the more flexible recommendation of the American Academy of Pediatrics (2011) of two hours of sedentary behaviour. Considering that preschool children included in this research exceed all given recommendations, there is a practical need to focus on decreasing their sedentary behaviour. They should be helped, both by their parents and teachers, to better organize their free 
time so that it is spent in active play.

Previous research shows a decline in physical activity with the increasing age (Jurakic\& Haimer, 2012; Sallis et al., 2000). Therefore, particular attention should be given to preschool girls, who are lagging in physical activity compared to preschool boys and have a greater decline in physical activity than boys with increasing age (Cragss et al., 2011). According to the World Health Organization (WHO, 2018), a similar percentage of Croatian boys (89\%) and girls (87\%) aged 8 meet the recommended daily level of physical activity, while this percentage decreases dramatically by the age of 15 to $25 \%$ of adequately active boys and $12 \%$ of adequately active girls (WHO, 2018).

The main limitation of this study is that physical activity and sedentary behaviour due to the large sample of children were estimated using questionnaires filled out by the parents of preschool children. Future studies on physical activity and sedentary behaviour in preschool children should be done using more objective means of measuring activity, such as accelerometers. Such devices would provide better information about physical activity and its intensity level (low, moderate, vigorous activity) than any questionnaire, especially one not assessed by a person about whose physical activity we are interested. Furthermore, children spent part of weekdays in kindergarten. Parents are not present during that time in daycare, and there is a possibility of under- or overestimation of physical activity assessment by parents.

Other socioeconomic and environmental factors could be attributed to differences in physical activity and sedentary behaviour in the Mediterranean and continental regions. Further analysis of these factors could provide additional information to specific research areas and should be addressed in future studies.

The lower level of physical activity and a higher sedentary behaviour in the continental regions in comparison to the coastal regions is an issue that should be specifically addressed. There is a need for a specific intervention strategy for improving physical activity in preschool institutions in the continental parts of Croatia. We believe that continental preschool institutions could benefit greatly from the experiences of the educational workers and kinesiologists from the coastal macro-regions. Such an exchange of good practices should be stimulated.

\section{References}

American Academy of Pediatrics. (2011). Media use by children younger than 2 years. Pediatrics, 128(5), 10401045.https://doi.org/10.1542/peds.2011-1753

Barbosa, S.C., \& Oliveira, A.R. (2016). Physical activity of preschool children: A review. Journal of Physiotherapy of Physical Rehabilitation, 1(2). https://doi.org/10.4172/25730312.1000111

Bauman, A.E., Reis, R.S., Sallis, J.F., Wells, J.C., Loos, R.J.F., \& Martin, B.W. (2012). Correlates of physical activity: Why are some people physically active and others not? The Lancet, 380(9838), 258-271. https://doi.org/10.1016/ S0140-6736(12)60735-1

Bergman Markovic, B., Vrdoljak, D., Kranjcevic, K., Vucak, J., Kern, J., Bielen, I., Ivezic Lalic, D., Katic, M., \& Reiner, Z. (2011). Continental-Mediterranean and rural-urban differences in cardiovascular risk factors in Croatian population. Public Health, 52(4), 566-576. https://doi. org/10.3325/cmj.2011.52.566

Cardon, G., Van Cauwenberghe, E., Labarque, V., Haerens, L., \& De Bourdeaudhuij, I. (2008). The contribution of preschool playground factors in explaining children's physical activity during recess. International Journal of Behavioral Nutrition and Physical Activity, 5(11). https:// doi.org/10.1186/1479-5868-5-11

Craggs, C., Corder, K., Van Sluijs, E.M.F., \& Griffin, S.J. (2011). Determinants of change in physical activity in children and adolescents: A systematic review. American Journal of Preventive Medicine, 40(6), 645-658. https://doi. org/10.1016/j.amepre.2011.02.025

Culjak, Z., Miletic, D., Delas Kalinski, S., Kezic, A., \& Zuvela, F. (2014). Fundamental movement skills development under the influence of a gymnastics program and everyday physical activity in seven-year-old children. Iranian Journal of Pediatrics, 24(2), 124-130.

Department of Health and Aging. (2009). Get up and grow healthy eating and physical activity for early childhood: Staff and career book. Retrieved November 12, 2020, from https://www1.health.gov.au/internet/main/ publishing.nsf/Content/2CDB3A000FE57A4ECA257BF0001916EC/\$File/HEPA\%20-\%20B5\%20Book\%20-\%20 Staff\%20and\%20Carer\%20Book_LR.pdf

Department of Health, Physical Activity, Health Improvement and Protection. (2011). Start active, stay active: A report on physical activity from the four home countries' Chief Medical Officers. Retrieved November 12, 2020, from https://assets.publishing.service.gov.uk/government/ uploads/system/uploads/attachment_data/file/830943/ withdrawn_dh_128210.pdf

Finn, K., Johannsen, N., \& Specker, B. (2002). Factors associated with physical activity in preschool children. The Journal of Pediatrics, 140(1), 81-85. https://doi. org/10.1067/mpd.2002.120693

Hall, C.J.S., Eyre, E.L.J., Oxford, S.W., \& Duncan, M.J. (2018). Relationships between motor competence, physical activity, and obesity in British preschool aged children. Journal of Functional Morphology and Kinesiology, 3(4), 57. https://doi.org/10.3390/jfmk3040057

Investments that work for physical activity. (2012). British Journal of Sports Medicine. Retrieved November 12, 2020, from https://bjsm.bmj.com/content/bjsports/46/10/709. full.pdf

Janssen, I., Katzmarzyk, P.T., Boyce, W.F., Vereecken, C., Mulvihill, C., Roberts, C., Currie, C., \& Pickett, W. (2005). Comparison of overweight and obesity prevalence in school-aged youth from 34 countries and their relationships with physical activity and dietary patterns. Wiley Online Library, 6(2), 123-132. https://doi.org/10.1111/j.1467789X.2005.00176.x

Janz, K.F., Broffitt, B., \& Levy, S.M. (2005). Validation evidence for the Netherlands Physical Activity Questionnaire for young children: The Iowa Bone Development Study. Research Quarterly for Exercise and Sport, 76(3), 363-369. https://doi.org/10.1080/02701367.2005.10599308

Jurakic, D., \& Heimer, S. (2012). Prevalence of insufficient physical activity in Croatia and in the World: An overview of studies. Arhiv za higijenu rada i toksikologiju, 63(3), 3-12. Retrieved November 12, 2020, from https://hrcak. srce.hr/92072

Jurakic, D., \& Pedisic, Z. (2019). Croatian 24-hour guidelines 
for physical activity, sedentary behaviour, and sleep: A proposal based on a systematic review of literature. Retrieved November 12, 2020, fromhttps://hrcak.srce.hr/ index.php?show=clanak\&id_clanak_jezik=330709

Kezic, A., \& Miletic, D. (2014). Does physical activity affect specific rhytmic gymnastics skills in children? Paper presented at The 8th International Scientific and Professional Conference A Child in Motion in the Koper, Slovenia. Retrieved January 5, 2020, from https://issuu. com/aplikativnakineziologijaprivat/docs/proceedings_ book_a_child_in_motion_/3

Lavie, C.J., Ozemek, C., Carbone, S., Katzmarzyk, P.T., \& Blair, S.N. (2019). Sedentary behavior, exercise, and cardiovascular health. Circulation Research, 124(5), 799815. https://doi.org/10.1161/CIRCRESAHA.118.312669

Petric, V., Holik, I., Blazevic, I., \& Vincetic, N. (2019). Correlation between the education of parents and toddlers about the importance of movement and level of physical activity. Retrieved January 5, 2020, from https://www.bib. irb.hr/1022052

Sallis, J.F., Prochaska, J.J., \& Taylor, W.C. (2000). A review of correlates of physical activity of children and adolescents. Medicine and Science in Sports and Exercise, 32(5), 963975. https://doi.org/10.1097/00005768-200005000-00014

Santaliestra-Pasias, A.M., Mouratidou1, T., Verbestel, V., Bammann, K., Molnar, D., Sieri, S., Siani, A., Veidebaum, T., Marild, S., Lissner, L., Hadjigeorgiou, C., Reisch, L., De Bourdeaudhuij, I., \& Moreno, L.A. (2013). Physical activity and sedentary behaviour in European children: The IDEFICS study. Public Health Nutrition, 17(10), 2295 2306. https://doi.org/10.1017/S1368980013002486

Tremblay, M.S., LeBlanc, A.G., Carson, V., Choquette, L., Connor Gorber, S., Dillman, C., Duggan, M., Gordon, M.J., Hicks, A., Janssen, I., Kho, M.E., Latimer-Cheung, A.E., LeBlanc, C., Murumets, K., Okely, A.D., Reilly, J.J., Spence, J.C., Stearns, J.A., \& Timmons, B.W. (2012). Canadian physical activity guidelines for the early years (aged 0-4 years) [Electronic version]. Applied Physiology, Nutrition, and Metabolism, 37(2). https://doi.org/10.1139/ h2012-018

Tucker, P. (2008). The physical activity levels of preschool children: A systematic review. Early Childhood Research Quarterly,23(4), 547-558. https://doi.org/10.1016/j. ecresq.2008.08.005

World Health Organization. (2018). Croatia physical activity factsheet 2018. Retrieved January 5, 2020, from http:// www.euro.who.int/_data/assets/pdf_file/0004/382342/ croatia-eng.pdf

World Health Organization. (2019). To grow up healthy, children need to sit less and play more: New WHO guidelines on physical activity, sedentary behaviour and sleep for children under 5 years of age. Retrieved January 5, 2020, from https://apps.who.int/iris/ bitstream/handle/10665/311664/9789241550536-eng. pdf? sequence $=1$ \&isAllowed $=y$ 